\title{
Membrane-binding Peptides for Extracellular Vesicles on-chip Analysis
}

Alessandro Gori*1, Alessandro Romanato ${ }^{1}$, Greta Bergamaschi ${ }^{1}$, Alessandro Strada ${ }^{1}$, Paola Gagni ${ }^{1}$, Roberto Frigerio ${ }^{1}$, Dario Brambilla ${ }^{1}$, Riccardo Vago $^{2}$, Silvia Galbiati ${ }^{2}$, Silvia Picciolini ${ }^{3}$, Marzia Bedoni $^{3}$, George G. Daaboul ${ }^{4}$, Marcella Chiari ${ }^{1}$ and Marina Cretich*1

1: Consiglio Nazionale delle Ricerche, Istituto di Chimica del Riconoscimento Molecolare (ICRM), Milano, Italy

2: San Raffaele Scientific Institute, Milano, Italy

3: Laboratory of Nanomedicine and Clinical Biophotonics (LABION), IRCCS Fondazione Don

Carlo Gnocchi, Milano, Italy

4: NanoView Biosciences, Boston, MA, USA

*Corresponding authors: alessandro.gori@cnr.it, marina.cretich@cnr.it

\begin{abstract}
Small extracellular vesicles (EVs) presents fairly distinctive lipid membrane features in the extracellular environment. These include high curvature, lipid packing defects and a relative abundance in lipids such as phosphatidylserine and ceramide. EVs membrane could be then considered as a 'universal' marker, alternative or complementary to traditional characteristic surfaceassociated proteins.

Here we introduce the use of membrane sensing peptides as new, highly efficient ligands for EVs capturing onto bioanalytical chips. In particular, we took advantage of bradykinin-derived peptidic baits to directly integrate EVs capturing and analysis on a microarray platform, even using serum without pre-isolation steps. EVs were analyzed by label-free, single particle counting and sizing, and by fluorescence co-localization immune staining with fluorescent anti-CD9/anti-CD63/antiCD81antibodies.

Peptides performed as selective yet general EVs baits and showed a binding capacity higher than antitetraspanins antibodies. Insights into surface chemistry for optimal peptide performances are also discussed, as capturing efficiency is strictly bound to probes surface orientation and multivalency effects. We anticipate that this new class of ligands, also due to the versatility and limited costs of synthetic peptides, may greatly enrich the molecular toolbox for EVs analysis.
\end{abstract}

Keywords: Extracellular Vesicles; Peptides; Microarrays; Membrane Binding; Membrane Curvature 


\section{Introduction}

Extracellular vesicles (EVs) are membranous micro- and nano-sized biological particles released by cells that play a major role in inter-cellular communication. EVs shuttle an impressive amount of molecular information, including proteins and non-coding RNAs, thus representing a phenomenal source of circulating biomarkers [1]. As such, EVs are arising unparalleled expectations as the next generation theranostic tools [2]. However, to fully realize EVs potential, several challenges in their separation and analysis are yet to be overcome [3].

Indeed, some EVs physicochemical features (e.g. size, buoyant density) overlap those of other nanoscale components of biological fluids, such as high-density lipoproteins (HDLs), viruses, organelles and protein aggregates. These nano-sized contaminants can be accidentally co-isolated with EVs, affecting the downstream analysis of vesicles with regards to their count, function and content [4]. Analytical platforms for EVs high-throughput analysis that do not strictly rely on sample pre-treatment, limiting purification artifacts, are therefore highly desirable.

In this scenario, EV microarrays have been introduced by Jørgensen and collaborators to phenotype EVs on a protein microarray platform [5]. In this technique, antibodies are used for the selective capturing of EVs by their surface-associated proteins, followed by EVs detection via fluorescencebased immune-staining of characteristic trans-membrane proteins (CD9, CD63 and CD81). Of note, this approach does not require prior EVs isolation but can rather be performed directly on complex biological samples. This format has been extended to the analysis of antibody captured vesicles in a label free mode using Surface Plasmon Resonance Imaging (SPRi) [6] and Single Particle Interferometric Reflectance Imaging Sensor (SP- IRIS) [7]. Commonly used markers for EV capturing include tetraspanins (CD9, CD63, CD81, CD82), MHC class I and II, HSP70, Annexin V, Flotillin and EpCAM. However, targeting surface-exposed proteins still present several drawbacks: i) the analysis can be biased by the presence of soluble antigens; ii) the inherent variability of antibody specificity and affinity can impair EVs capturing efficiency; iii) protein markers relative abundance may be poor or subject to significant inter-individual fluctuations, thus reducing the value of comparative studies.

The possibility to target a specific but "universal” EVs marker such as the lipid membrane would therefore represent a paradigmatic shift, possibly expanding the available molecular tools towards an increased analytical consistence.

In this regard, EVs membrane is characterized by physical and chemical traits that are peculiar in the extracellular space [8]. Small EVs have indeed highly curved membranes, whose outer leaflets typically contain a high amount of anionic, unsaturated phospholipids (e.g. phosphatidylserine) together with the presence of characteristic lipid packing defects [9], [10], [11].

Of note, many proteins are physiologically involved in the dynamic modulation of membrane curvature that occurs during a multitude of cellular processes (including vesicles secretion); in addition it is further worth highlighting that some of them are able to sense and bind with exquisite selectivity only highly curved membranes [12], [13], [14], [15]. These include, among others, the Bin-Amphiphysin-Rvs (BAR) domain of ampiphysin [16], the ArfGAP1 Lipid Packing Sensor (ALPS) proteins [17], the C2B domain of Synaptotagmin-I and the effector domain of the myristoylated alanine-rich C-kinase substrate protein (MARCKS-ED) [18]. Accordingly, peptides derived from membrane sensing proteins have emerged as convenient, easy-to-synthetize novel molecular probes for targeting highly curved membranes [11], [19], [20], [21]. In this frame, proposed mechanisms of membrane curvature sensing by protein domains and peptides can be multiple and cooperative (Figure 1). In many cases, the early events of membrane recognition and binding are based on complementary electrostatic interactions between the peptide/protein effector domain and the phospholipids on the outer membrane leaflet, that subsequently can lead to the insertion of the sensing effector into the membrane defects that characterize highly curved membranes [18],[21], [13]. This mechanism is 
characteristic of amphipathic peptides. Other recognition pathways are characterized by a structural complementarity where curved protein scaffolds match the curvature of membranes, or entail the possibility that membrane curvature recognition occurs as a result of entropy-driven phenomena [22]. Lipid affinity specific interactions were also reported. Overall, all the factors involved in membrane recognition remain complex and likely to be still fully elucidated. Notwithstanding this, the use of membrane sensing peptides is arising an increasing interest, due to the ease of synthesis and chemical manipulation and low-cost of production.

A

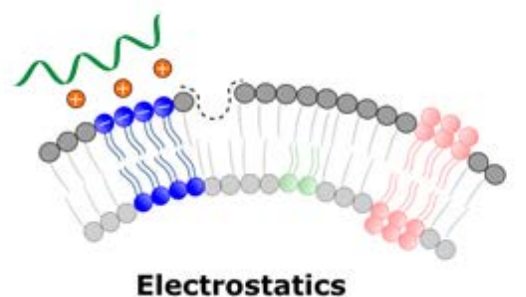

B

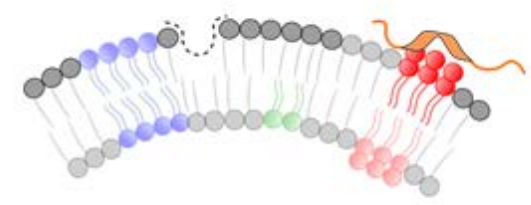

Lipid binding

C

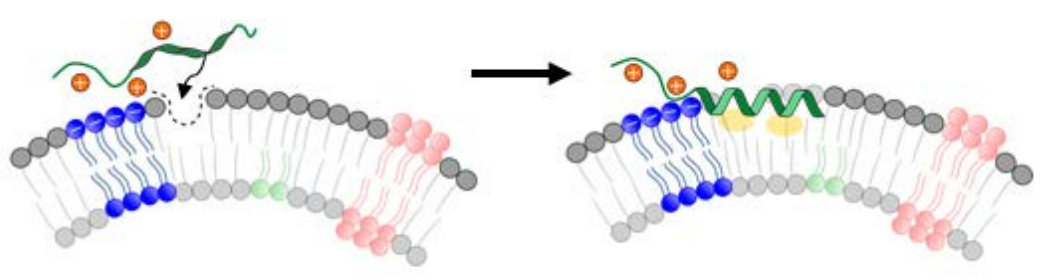

Amphipathic peptides

Figure 1. Common mechanisms involved in membrane recognition by curvature sensing peptides. A) purely electrostatic interactions are typical of cationic peptides; B) specific binding to lipids particularly abundant in small vesicles (e.g. phosphatidylserine) can drive the interaction; C) amphipathic peptides usually approach highly curved membranes through electrostatics, and subsequently insert into lipid packing defects. Binding can be stabilized by peptide folding within the membrane, facilitated by the presence of hydrophobic groups.

Here, we introduce for the first time the use of membrane-sensing peptide ligands as molecular baits for small EVs and we demonstrate their use in an EVs microarray platform as valuable alternative/complement to antibodies. The peptide ligands were able to capture EVs from purified samples and from untreated human serum with high specificity and binding capacity.

To the best of our knowledge, this is the first application of membrane sensing peptides to EVs profiling and the first reported example of EVs peptide microarrays. 


\section{Results and discussion}

\subsection{Peptide design and microarray}

Among the set of reported curvature-sensing peptides, we decided to focus on a short amino acid sequence (RPPGFSPFR, BK) derived from Bradykinin that was reported to bind in solution to highly curved vesicle surfaces, particularly when displayed in a multivalent trimeric form (BK-tri). Due to the presence of two arginine residues and two phenylalanines along the sequence, an electrostaticsdominated recognition further stabilized by hydrophobic interactions has been proposed to be responsible for affinity to highly curved membranes [23].

We then adapted the reported molecule to fit our previously developed microarray platform for siteselective oriented peptide immobilization [24]. Briefly, the membrane sensing sequence was extended with a short PEG spacer terminating with a propargyl group for click-based bioconjugation to azide copolymer (MCP-6) coated analytical surfaces [25]. We realized three different peptide baits, including a linear (BP), a branched $(\mathrm{BPb})$ and a tandem $(\mathrm{BPt})$ derivatives, to assess the possible contribution of probes multivalency to EVs capturing (Scheme 1).

Finally, given the key role of electrostatic interactions in initiating the complex membrane-sensing mechanism reported for BK, we also synthesized a negative control peptide where arginine residues were mutated to (oppositely charged) glutamic acid residues (BPn).

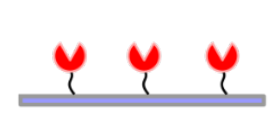

Clickable chip

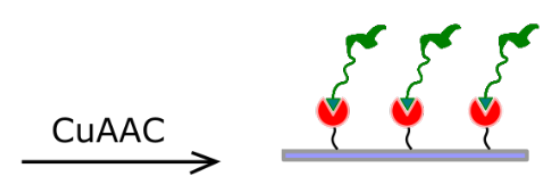

Bp linear

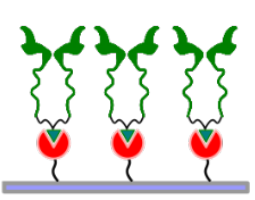

Bp branched

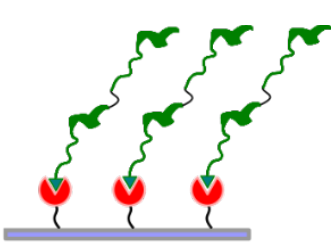

Bp tandem

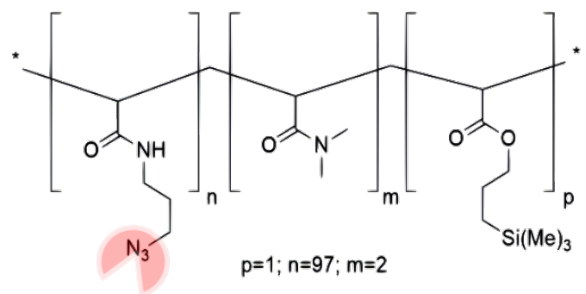

MCP-6 copolymer
BP: RPPGFSPFR-K-G-(O $\left.\mathrm{O}_{2} \mathrm{Oc}\right)_{2}-\mathrm{Prg}$

BPb: (RPPGFSPFR) $)_{2}-\mathrm{K}-\mathrm{G}-\left(\mathrm{O}_{2} \mathrm{Oc}\right)_{2}-\mathrm{Prg}$

BPt: RPPGFSPFR-(O $\left.\mathrm{O}_{2} \mathrm{Oc}\right)-\mathrm{RPPGFSPFR}-\mathrm{K}-\mathrm{G}-\left(\mathrm{O}_{2} \mathrm{Oc}\right)_{2}-\mathrm{Prg}$

BPn: EPPGFSPFE-K-G-(O $\left.\mathrm{O}_{2} \mathrm{Oc}\right)_{2}-\mathrm{Prg}$

Scheme 1: membrane sensing peptides are used to capture EVs on sensing surfaces. Peptidic probes are immobilized on chips through chemoselective click type reaction between azido groups provided by MCP-6 surface coating and propargyl glycine terminated peptides. Peptidic probes are synthesized in a linear form (BP) and in two multivalent presentation: branched $(\mathrm{BPb})$ and tandem $(\mathrm{BPt})$. As a negative control $(\mathrm{BPn})$, a peptide where arginine residues were mutated to (oppositely charged) glutamic acid residues was synthesized

\subsection{EVs capturing on peptide microarray}

Patterned silicon chips with $80 \mathrm{~nm} \mathrm{SiO}$ layer, suitable for SP-IRIS by the ExoView ${ }^{\mathrm{TM}}$ platform, were arrayed with 5 replicated spots of peptides via copper catalyzed azide-alkyne cycloaddition (CuAAC) mediated chemoselective immobilization (Scheme 1). Microchips were first characterized by 
quantifying the immobilization density of peptides; according to our previous findings [24], [26], the average binding yield of each spotted peptide was $2.0-3.5 \mathrm{ng} / \mathrm{mm}^{2}$.

As a first experimental set to probe peptide-based EVs capturing, EVs were isolated from HEK cells by ultracentrifugation (UC) and characterized according to MISEV2018 guidelines [27], by Nanoparticle Tracking Analysis (NTA), Transmission Electron Microscopy (TEM) and Western blotting (WB) to demonstrate the presence of EV membrane and luminal proteins. Results confirmed the presence of vesicles with size, morphology and protein content compatible with EVs and are shown in the Supplementary Information (Figures S1-S3).

Based on NTA quantifications, serial dilutions of the HEK UC sample in the $1 \times 10^{6}-1 \times 10^{9}$ particles/mL range were prepared in PBS and incubated for 150 minutes on peptide chips. EV's capturing from the HEK UC sample on peptide microarray was analyzed by SP-IRIS on the ExoView $^{\mathrm{TM}}$ platform that digitally count, size and image individual, low-refractive index nanovesicles based on an interferometric principle [7] (Scheme 2); net values of detected EVs on BP and BPn from five spot replicates were averaged (Figure 2A).
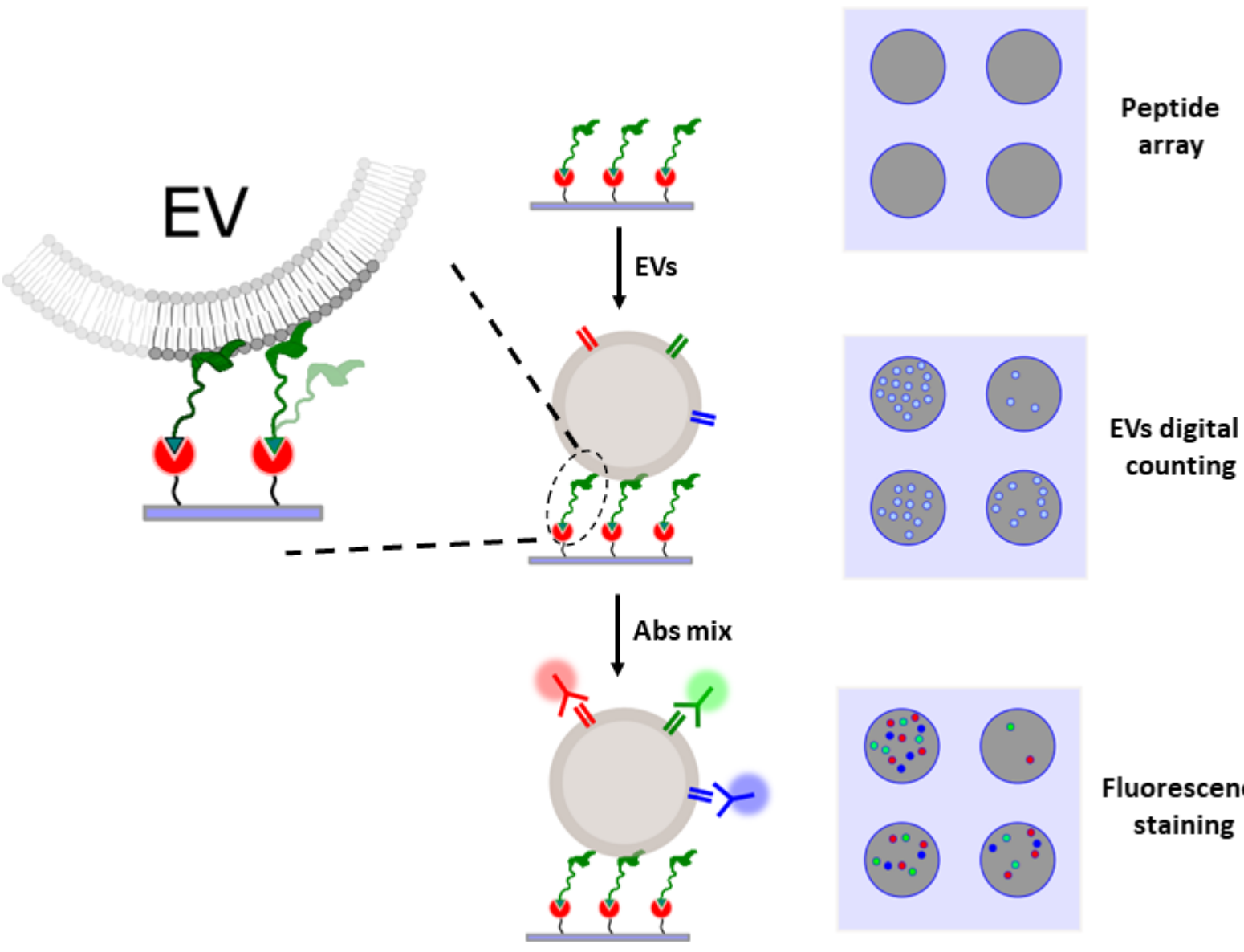
Fluorescence staining

Scheme 2. Scheme of the assay for label free and fluorescence detection of EVs captured on microarray chips. A silicon chip is arrayed with spots of capturing peptides and incubated with the EV sample. SP-IRIS platform images the chip and provides a label-free counting and sizing of the captured EVs. The same chip can then be further incubated with fluorescent antibodies for immune-staining of $\mathrm{EV}$ associated proteins and 3 colors fluorescence based co-localization of EV surface markers.

Remarkably, we could immediately assess that a significant amount of EVs (blue dots) could be detected within the area of BP spots (Figure 2B), whilst negligible binding was observed for the negative control peptide BPn. Moreover, microchips incubation with different sample concentrations 
highlighted a dose-response effect, with detected particle signal on BP being clearly distinguishable down to $1 \times 10^{7}$ particles/mL concentration (Figure 2A). It is worth noting that, when the same serial dilutions of the HEK UC sample $\left(1 \times 10^{6}-1 \times 10^{9}\right.$ particles/mL) were incubated on an antibody microarray chip spotted with anti-tetraspanins IgG (anti CD81/CD63/CD9), only the highest concentration $\left(1 \times 10^{9}\right.$ particles $\left./ \mathrm{mL}\right)$ provided a distinguishable counting from the non-specific signals detected on the negative antibody control (Figure 2C). Of note, the average particle counting detected on the antibody spots was lower than that provided by BP for each tested HEK UC sample. As for the multivalent peptide forms $\mathrm{BPb}$ and $\mathrm{BPt}$, we observed only a slight increase in vesicle binding (Figure 2D). In this sense, a surface multivalency effect due to peptides cooperative interaction in nanovesicles binding is likely to account for the observed high capturing efficiency (Scheme 2). Representative spot images for the whole set of peptides are reported in the Supplementary Information (Figure S4).

A

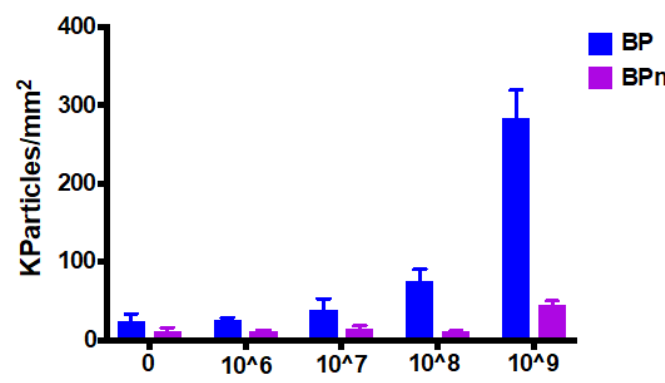

C

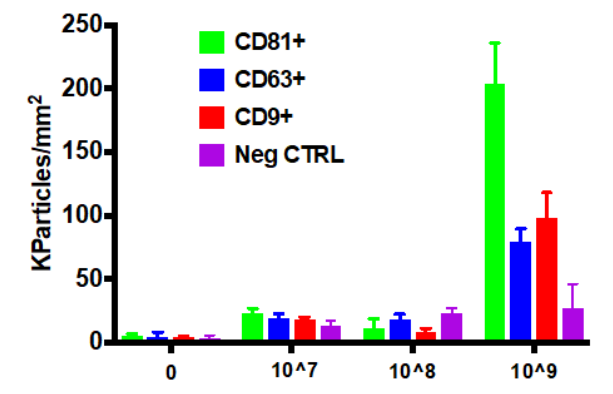

B

BP
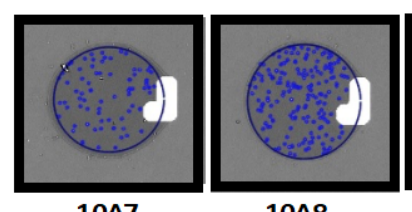

$10^{\wedge} 8$

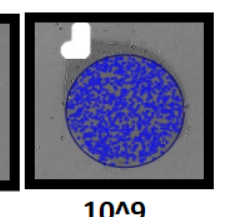

BPn

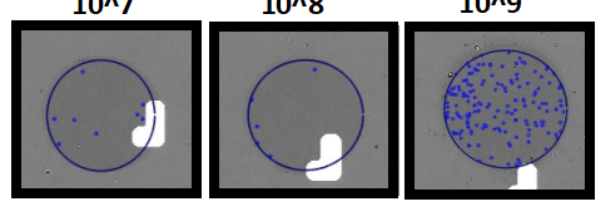

D

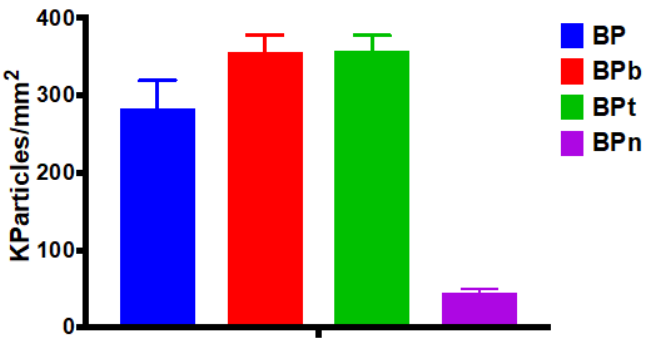

Figure 2: A) HEK UC particle density per $\mathrm{mm}^{2}$ detected on BP and BPn peptide spots in a blank sample (filtered PBS) and in $1 \times 10^{6}-1 \times 10^{9}$ particles/mL concentrations range. A clear dose-response effect is visible. Signal on BPn peptide is negligible. B) Representative images of BP and BPn peptide spots incubated with $1 \times 10^{7}-1 \times 10^{9}$ particles $/ \mathrm{mL}$ : blue dots indicate detected particles. C) HEK UC particle density per $\mathrm{mm}^{2}$ detected on antibody microarray (anti CD81/CD63/CD9). Only $1 \times 10^{9}$ particles/mL concentration provides on $\mathrm{CD}$ antibodies spots a signal distinguishable from that on the negative control antibody. D) Particle density per $\mathrm{mm}^{2}$ detected on BP and multivalent BPb and BPt peptides when incubated with $1 \times 10^{9}$ particles/mL HEK UC sample. Only a slight increase in vesicle binding capacity is provided by multivalency. Representative peptide spot images are reported in the Supplementary Information (Figure S4)

To confirm the EV nature of the peptide captured vesicles, we performed a 3 colors fluorescence detection of EV transmembrane proteins (CD81, CD63, CD9) by the use of a secondary antibody staining with a cocktail of anti- CD81, anti-CD9 and anti-CD63 antibodies labelled with AF555, AF488 and AF647 respectively. When HEK UC sample at the concentration of $2 \times 10^{9}$ particles $/ \mathrm{mL}$ was incubated on peptide chip, label free counting (Figure 3A) and bright fluorescence signals were detected on particles captured by BP peptides in the three fluorescence channels (Figure 3B and C). The co-localization experiment confirmed that vesicles captured on BP spots express on their surface the three tetraspanin proteins that are considered typical markers of EVs [27]. In accordance to label free data, the negative control BPn showed negligible non-specific binding (Figure 3A and B). 
A

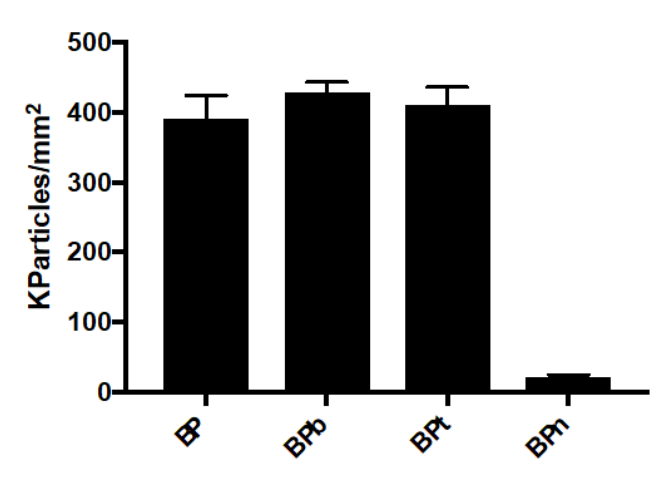

C

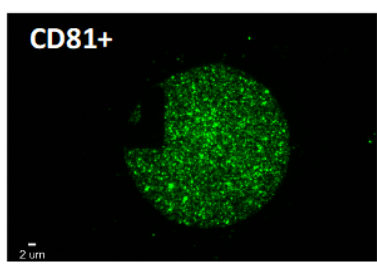

B
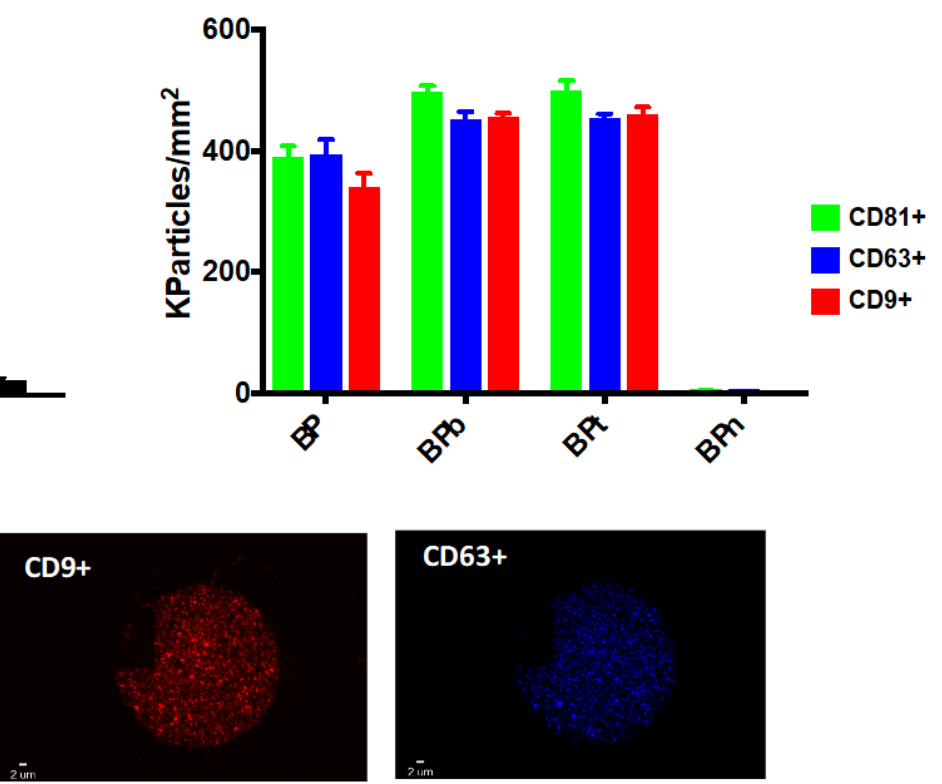

CD63+

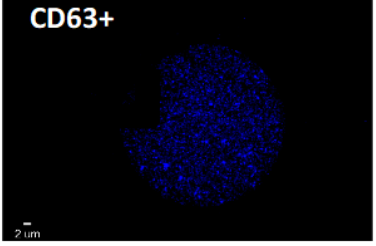

Figure 3: A) EV density after incubation with HEK UC sample at the concentration of $2 \times 10^{9}$ particles/mL label free detected on BP peptides; B) Correspondent EV density detected by fluorescence on BP peptides; C) Representative BP spot and fluorescence immune-staining. Images were acquired on the three different fluorescence channels: green particles are vesicles captured by BP and positive for CD81; blue particles are vesicles captured by BP and positive for CD63 whereas red particles are vesicles captured by BP and positive for CD9.

\subsection{EVs from human serum}

In order to test our peptide arrays with samples of increasing complexity, capturing of EVs from human serum by membrane-sensing peptides was assessed with vesicles derived from a pool of serum samples and isolated by two common procedures: ultracentrifugation (UC) and Size Exclusion Chromatography (SEC). Isolated samples were characterized by NTA, TEM and WB (Supplementary Information and Figures S5-S19). Label free counting on BP peptides and fluorescence colocalization of tetraspanins were performed on both UC (Figure 4A) and SEC isolated vesicles (Figure 4B) demonstrating wide applicability of BP ligands with different samples and purification protocols. Differently from what observed with the HEK derived vesicles, the multivalent $\mathrm{BPb}$ and BPt peptides showed an improved capturing capacity of serum derived particles in comparison to linear BP (Figure 4A-B). Importantly, co-localization detection of EV transmembrane proteins (CD81, CD63, CD9) confirm the capturing of EV by the entire set of peptides except for the negative control and show the same trend of increased binding yield for multivalent peptides observed by label-free counting.

Given the relevance of isolation-free workflows to obtain unbiased EV analysis, in addition to purified vesicles, peptide ligands were tested with pure serum (Figure 4C). A pooled sera sample was filtered by a $0.2 \mu \mathrm{m}$ syringe filter, diluted 1:8 in PBS and incubated on a peptide arrayed chip for 150 min. Particles from untreated serum were label free detected with similar efficiency on all peptides except for the negative control BPn (Figure 4C). Remarkably, fluorescence staining with antiCD9/CD63/CD81 antibodies revealed a clear effect of peptide multivalency in capturing a higher number of tetraspanin positive particles compared to linear BP (Figure 4C). 
These findings confirm that the use of multivalent peptides can be particularly convenient to increase affinity and capturing specificity with complex samples such as serum. This is not surprising, as a multivalency-enhanced nanovesicles recognition for Bradykinin was already described by Yin and co-workers [23], and we previously reported on multivalency favorable role in peptide microarrays [24].

Specific EVs binding on BP peptides directly from untreated serum was further demonstrated by a widely used and independent detection technique using a Surface Plasmon Resonance Imaging (SPRi) assay. To this aim, unpurified serum diluted 1:10 was injected on an SPRi chip arrayed with the set of BP peptides according to previously devised protocols [24] and as described in the experimental section. EV binding was detected label free and then the effective immobilization of EVs on the surface of SPRi chip was confirmed by injecting a mixture of anti-CD9/CD63/CD81 antibodies that interact with surfaces of EVs detected on BP peptides (Figure S10).

A
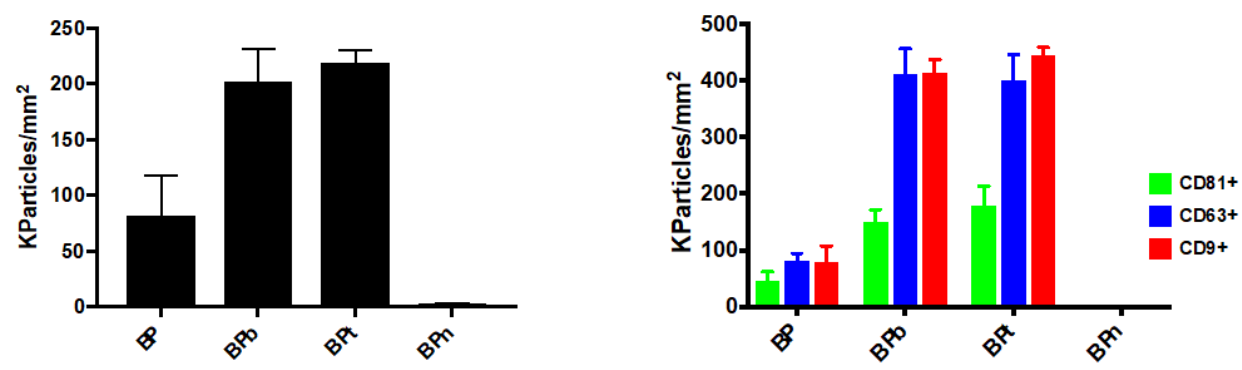

B
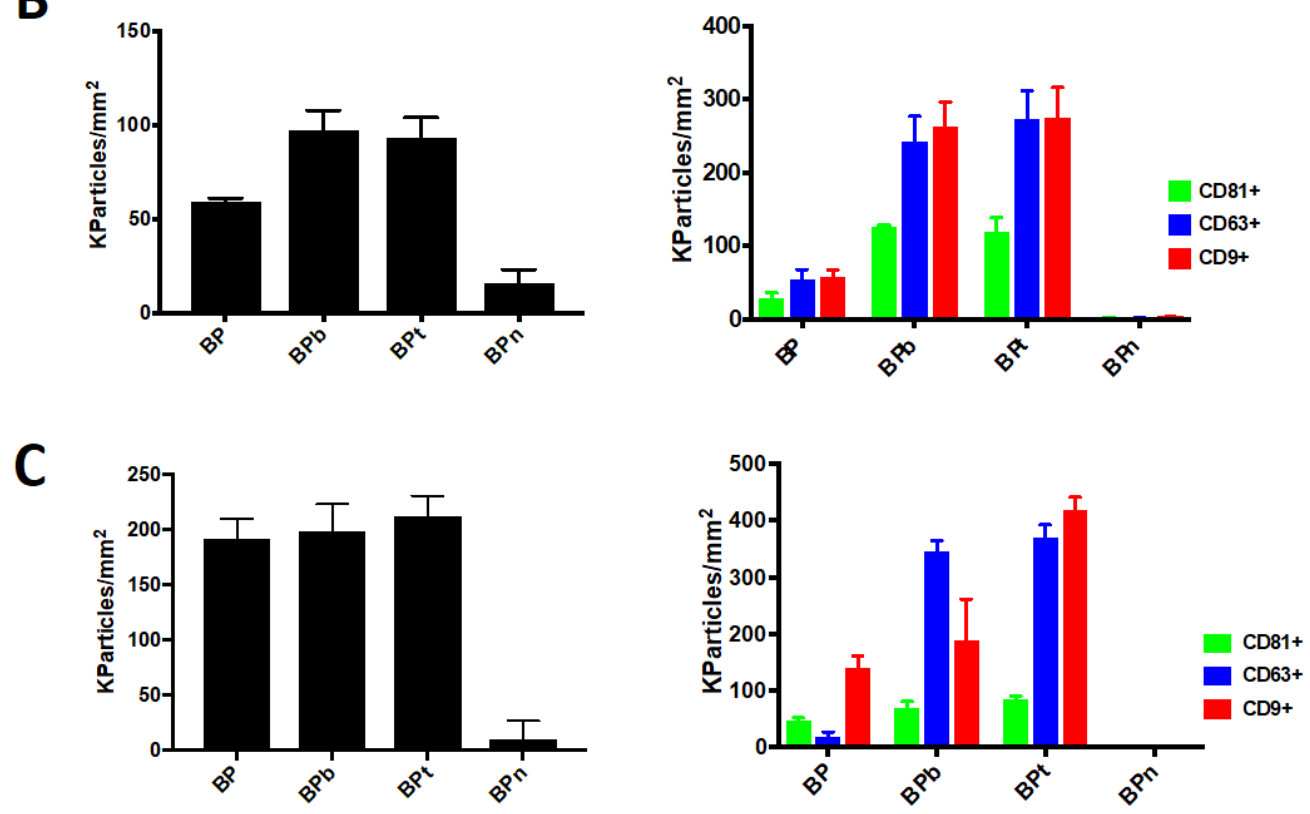

Figure 4: A): Analysis of EV isolated by ultracentrifugation from human serum incubated on peptide microarrays at $1 \times 10^{9}$ particles/mL concentration. Density of particles captured by BP peptides (left panel) is confirmed by fluorescence staining using CD81/CD/63/CD9 fluorescent antibodies (right panel). B): Density of EVs isolated by SEC from human serum incubated on peptide microarrays at $1.27 \times 10^{8}$ particles $/ \mathrm{mL}$ concentration and captured by BP peptides (left panel), confirmed by fluorescence staining using CD81/CD/63/CD9 fluorescent antibodies (right panel). C): Analysis performed 
on unpurified human serum diluted 1:8. Density of particles captured by BP peptides is detected label free (left panel) and by fluorescence staining using CD81/CD/63/CD9 fluorescent antibodies (right panel)

\subsection{The role of surface chemistry in vesicles binding}

To assess the role of peptide exposure and spatial orientation upon chip surface on the efficiency of EVs capturing, we compared a nonspecific immobilization on nucleophile-reactive polymer-coated chips $v s$ a click-type peptides binding strategy able to provide predetermined probes orientation. Of note, both strategies rely on the same polymer for chips coating (MCP-2) [28], that can be differently functionalized to introduce azido reactive handles (MCP-6) [25].

Peptide microarrays were incubated with SEC isolated EVs from serum (1x10 ${ }^{9}$ particles/mL ). Strikingly, for the entire set of BP peptides, the EV binding capacity observable for site-selectively immobilized peptides was totally abolished when peptides were randomly bound onto the surface (Figure 5A and 5B). To provide additional insights on the general feasibility of our approach, peptide surface orientation was also pursued by means of the well-known biotin-streptavidin system, that though less efficient than click-type immobilization, maintained EVs binding capacity (Supplementary Information Figure S11).

A

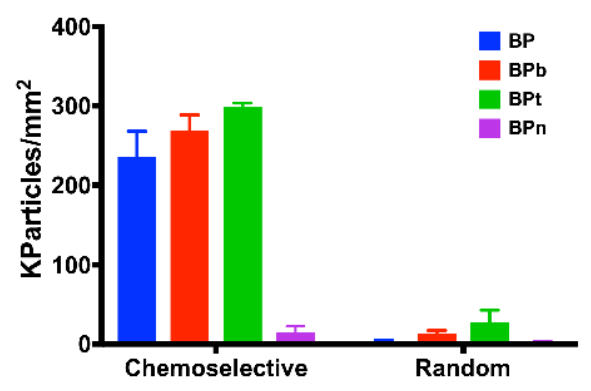

B

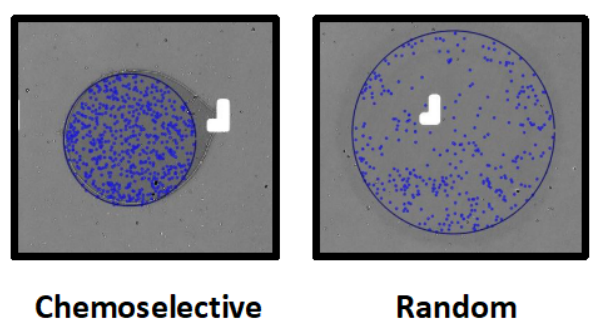

Figure 5 A): EV density on BP peptides immobilized either chemoselectively on MCP-6 or randomly on MCP-2. EV capturing capacity is abolished when peptides are not oriented on the microarray surface. B): comparison of representative images of BP spots either chemoselectively or randomly immobilized. Spot size is smaller on MCP-6, edges well defined and particle counting after incubation higher than on the random bound peptide.

\subsection{Vesicles Binding on BP peptides is not mediated by surface associated proteins}

In order to get insights into the EV-peptide binding mechanism and verify whether it is directly mediated by the lipid membrane or influenced by its associated proteins, serum EVs isolated by SEC were subjected to trypsin digestion [29], characterized by NTA (Supplementary information Figure S12) and incubated at the concentration of $1 \times 10^{9}$ particles $/ \mathrm{mL}$ on peptide microarrays (Figure 6A). Particle density of the trypsin-treated sample detected on BP spots compared to that of a sample subjected to analogous incubation in absence of proteolytic enzyme, demonstrated that membrane protein digestion does not affect vesicles binding. Indeed, binding in some cases (namely with the multivalent peptides) is even enhanced by proteolysis thus not being directly mediated by surface associated proteins. Oppositely, when the same samples were incubated on an antibody (CD81/CD63/CD9) microarray, as expected, no binding was detectable with the trypsin digested sample likely due to proteolysis of EV surface tetraspanins (Figure 6B). 
A

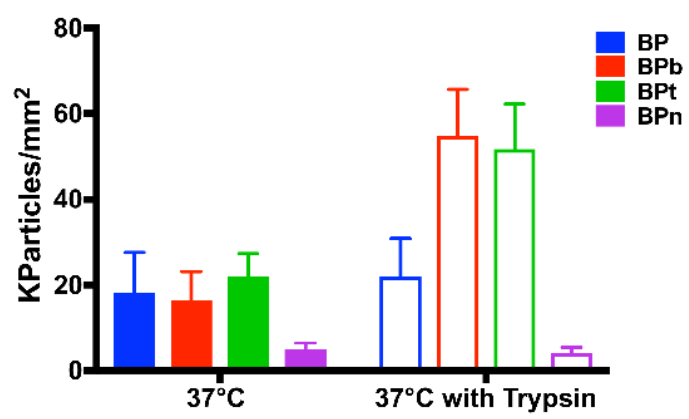

B

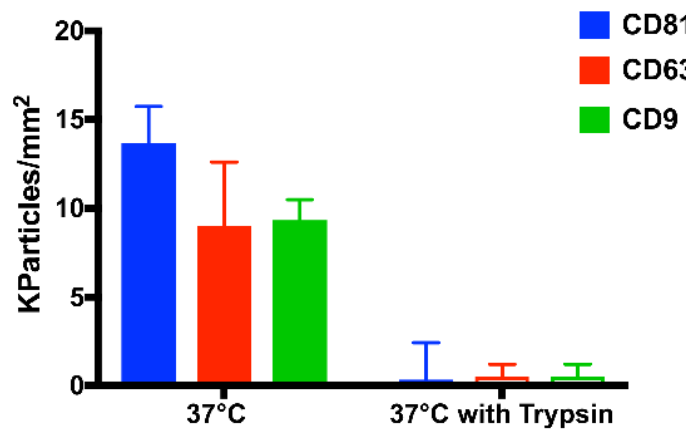

Figure 6: A): Particle density of SEC isolated EVs from human serum incubated at the concentration of $1 \times 10^{9}$ particles/mL. EVs capturing by BP peptides is not affected by trypsin treatment. (B) EVs binding on CD81/CD63/CD9 antibody chip is abolished by surface protein digestion using trypsin.

\section{Conclusions}

We here provided a proof-of-concept demonstration of the use of membrane sensing peptides as a novel class of molecular ligands for integrated EVs isolation and analysis, reporting for the first time on peptide microarrays for extracellular vesicles. Given their affinity to the EVs membrane, these molecules can serve as general baits, enabling vesicles capturing unbiased by differential surface protein expression. We showed that highly efficient EVs capturing can be obtained, even from unpurified and complex samples such as serum, provided the surface chemistry of peptidic probes presentation is accurately tailored. These new class of molecular probes may be nicely integrated with the use of protein markers towards improved EVs isolation and characterization. Of note, compared to proteins and antibodies, peptides are characterized by low cost of preparation, remarkable stability and ease of chemical manipulation, offering virtually unlimited possibilities for experimental design. We therefore envision these molecular tools to find broad applications within the EVs scenario in the near future.

\section{Acknowledgements}

HYDROGEX (Regione Lombardia\&Fondazione Cariplo, grant n. 2018-1720) and INDEX (European Union's Horizon 2020 research and innovation programme under grant agreement $\mathrm{N}^{\circ}$ 766466) projects are acknowledged for partial financial support to A.G., M.C. and M.C.; PRIN2015JTL4HL is also acknowledged for financial support to A.S.

\section{Notes}

The authors declare the following competing financial interest(s): Alessandro Gori, Marina Cretich and Marcella Chiari have filed a US Provisional Patent Application $(62 / 897,042)$ on the bases of these data.

\section{References}

[1] van Niel, G.; D’Angelo, G.; Raposo, G.: Shedding light on the cell biology of extracellular vesicles. Nature Reviews Molecular Cell Biology, 19, 213-228 (2018). http://doi:10.1038/nrm.2017.125.

[2] EL Andaloussi, S.; Mäger, I.; Breakefield, X. O.; Wood, M. J. a: Extracellular vesicles: 
biology and emerging therapeutic opportunities. Nature reviews. Drug discovery, 12, 347-57 (2013). http://doi:10.1038/nrd3978.

[3] Konoshenko, M. Y.; Lekchnov, E. A.; Vlassov, A. V.; Laktionov, P. P.: Isolation of Extracellular Vesicles: General Methodologies and Latest Trends. BioMed Research International, 2018, 1-27 (2018). http://doi:10.1155/2018/8545347.

[4] Merchant, M. L.; Rood, I. M.; Deegens, J. K. J.; Klein, J. B.: Isolation and characterization of urinary extracellular vesicles: Implications for biomarker discovery. Nat. Rev. Nephrol. (2017), 13, 731-749

[5] Jørgensen, M.; Bæk, R.; Pedersen, S.; Søndergaard, E. K. L.; Kristensen, S. R.; Varming, K.: Extracellular Vesicle (EV) Array: microarray capturing of exosomes and other extracellular vesicles for multiplexed phenotyping. Journal of extracellular vesicles, 2, 1-9 (2013). http://doi:10.3402/jev.v2i0.20920.

[6] Rojalin, T.; Phong, B.; Koster, H. J.; Carney, R. P.: Nanoplasmonic Approaches for Sensitive Detection and Molecular Characterization of Extracellular Vesicles. Frontiers in Chemistry, 7 (2019). http://doi:10.3389/fchem.2019.00279.

[7] Daaboul, G. G.; Gagni, P.; Benussi, L.; Bettotti, P.; Ciani, M.; Cretich, M.; Freedman, D. S.; Ghidoni, R.; Ozkumur, A. Y.; Piotto, C.; Prosperi, D.; Santini, B.; Ünlü, M. S.; Chiari, M.: Digital Detection of Exosomes by Interferometric Imaging. Scientific Reports, 6, 37246 (2016). http://doi:10.1038/srep37246.

[8] van Niel, G.; D’Angelo, G.; Raposo, G.: Shedding light on the cell biology of extracellular vesicles. Nature reviews. Molecular cell biology, (2018). http://doi:10.1038/nrm.2017.125.

[9] György, B.; Szabó, T. G.; Pásztói, M.; Pál, Z.; Misják, P.; Aradi, B.; László, V.; Pállinger, É.; Pap, E.; Kittel, Á.; Nagy, G.; Falus, A.; Buzás, E. I.: Membrane vesicles, current state-ofthe-art: Emerging role of extracellular vesicles. Cell. Mol. Life Sci. (2011), 68, 2667-2688

[10] Hugel, B.; Martínez, M. C.; Kunzelmann, C.; Freyssinet, J.-M.: Membrane Microparticles: Two Sides of the Coin. Physiology, 20, 22-27 (2005). http://doi:10.1152/physiol.00029.2004.

[11] Kastelowitz, N.; Yin, H.: Exosomes and microvesicles: Identification and targeting by particle size and lipid chemical probes. ChemBioChem (2014), 15, 923-928

[12] Lemmon, M. A.: Membrane recognition by phospholipid-binding domains. Nat. Rev. Mol. Cell Biol. (2008)

[13] Antonny, B.: Mechanisms of Membrane Curvature Sensing. Annual Review of Biochemistry, (2011). http://doi:10.1146/annurev-biochem-052809-155121.

[14] Cui, H.; Lyman, E.; Voth, G. A.: Mechanism of membrane curvature sensing by amphipathic helix containing proteins. Biophysical Journal, (2011). http://doi:10.1016/j.bpj.2011.01.036.

[15] Hatzakis, N. S.; Bhatia, V. K.; Larsen, J.; Madsen, K. L.; Bolinger, P. Y.; Kunding, A. H.; Castillo, J.; Gether, U.; Hedegård, P.; Stamou, D.: How curved membranes recruit amphipathic helices and protein anchoring motifs. Nature Chemical Biology, (2009). http://doi:10.1038/nchembio.213.

[16] Bhatia, V. K.; Madsen, K. L.; Bolinger, P. Y.; Kunding, A.; Hedegard, P.; Gether, U.; Stamou, D.: Amphipathic motifs in BAR domains are essential for membrane curvature sensing. EMBO Journal, (2009). http://doi:10.1038/emboj.2009.261.

[17] Bigay, J.; Casella, J. F.; Drin, G.; Mesmin, B.; Antonny, B.: ArfGAP1 responds to membrane curvature through the folding of a lipid packing sensor motif. EMBO Journal, (2005). http://doi:10.1038/sj.emboj.7600714.

[18] De Jesus, A. J.; White, O. R.; Flynn, A. D.; Yin, H.: Determinants of Curvature-Sensing Behavior for MARCKS-Fragment Peptides. Biophysical Journal, 110, 1980-1992 (2016). http://doi:10.1016/j.bpj.2016.04.007.

[19] Flynn, A. D.; Yin, H.: Lipid-Targeting Peptide Probes for Extracellular Vesicles. Journal of Cellular Physiology, 231, 2327-2332 (2016). http://doi:10.1002/jcp.25354. 
[20] Gómez-Llobregat, J.; Elías-Wolff, F.; Lindén, M.: Anisotropic Membrane Curvature Sensing by Amphipathic Peptides. Biophysical Journal, 110, 197-204 (2016). http://doi:10.1016/j.bpj.2015.11.3512.

[21] De Jesus, A. J.; Yin, H.: Computational design of membrane curvature-sensing peptides. In Methods in Molecular Biology; (2017); Vol. 1529, pp. 417-437

[22] Zeno, W. F.; Thatte, A. S.; Wang, L.; Snead, W. T.; Lafer, E. M.; Stachowiak, J. C.: Molecular Mechanisms of Membrane Curvature Sensing by a Disordered Protein. Journal of the American Chemical Society, 141, 10361-10371 (2019). http://doi:10.1021/jacs.9b03927.

[23] Saludes, J. P.; Morton, L. A.; Coulup, S. K.; Fiorini, Z.; Cook, B. M.; Beninson, L.; Chapman, E. R.; Fleshner, M.; Yin, H.: Multivalency amplifies the selection and affinity of bradykinin-derived peptides for lipid nanovesicles. Molecular BioSystems, (2013). http://doi:10.1039/c3mb70109c.

[24] Gori, A.; Cretich, M.; Vanna, R.; Sola, L.; Gagni, P.; Bruni, G.; Liprino, M.; Gramatica, F.; Burastero, S.; Chiari, M.: Multiple epitope presentation and surface density control enabled by chemoselective immobilization lead to enhanced performance in IgE-binding fingerprinting on peptide microarrays. Analytica Chimica Acta, 983, 189-197 (2017). http://doi:10.1016/j.aca.2017.06.027.

[25] Sola, L.; Damin, F.; Gagni, P.; Consonni, R.; Chiari, M.: Synthesis of Clickable Coating Polymers by Postpolymerization Modification: Applications in Microarray Technology. Langmuir, 32, 10284-10295 (2016). http://doi:10.1021/acs.langmuir.6b02816.

[26] Gori, A.; Sola, L.; Gagni, P.; Bruni, G.; Liprino, M.; Peri, C.; Colombo, G.; Cretich, M.; Chiari, M.: Screening Complex Biological Samples with Peptide Microarrays: The Favorable Impact of Probe Orientation via Chemoselective Immobilization Strategies on Clickable Polymeric Coatings. Bioconjugate Chemistry, 27, 2669-2677 (2016). http://doi:10.1021/acs.bioconjchem.6b00426.

[27] Théry, C.; Witwer, K. W.; Aikawa, E.; Alcaraz, M. J.; Anderson, J. D.; Andriantsitohaina, R.; Antoniou, A.; Arab, T.; Archer, F.; Atkin-Smith, G. K.; Ayre, D. C.; Bach, J.-M.; Bachurski, D.; Baharvand, H.; Balaj, L.; Baldacchino, S.; Bauer, N. N.; Baxter, A. A.; Bebawy, M.; Beckham, C.; Bedina Zavec, A.; Benmoussa, A.; Berardi, A. C.; Bergese, P.; Bielska, E.; Blenkiron, C.; Bobis-Wozowicz, S.; Boilard, E.; Boireau, W.; Bongiovanni, A.; Borràs, F. E.; Bosch, S.; Boulanger, C. M.; Breakefield, X.; Breglio, A. M.; Brennan, M. Á.; Brigstock, D. R.; Brisson, A.; Broekman, M. L.; Bromberg, J. F.; Bryl-Górecka, P.; Buch, S.; Buck, A. H.; Burger, D.; Busatto, S.; Buschmann, D.; Bussolati, B.; Buzás, E. I.; Byrd, J. B.; Camussi, G.; Carter, D. R.; Caruso, S.; Chamley, L. W.; Chang, Y.-T.; Chen, C.; Chen, S.; Cheng, L.; Chin, A. R.; Clayton, A.; Clerici, S. P.; Cocks, A.; Cocucci, E.; Coffey, R. J.; Cordeiro-da-Silva, A.; Couch, Y.; Coumans, F. A.; Coyle, B.; Crescitelli, R.; Criado, M. F.; D’Souza-Schorey, C.; Das, S.; Datta Chaudhuri, A.; de Candia, P.; De Santana, E. F.; De Wever, O.; del Portillo, H. A.; Demaret, T.; Deville, S.; Devitt, A.; Dhondt, B.; Di Vizio, D.; Dieterich, L. C.; Dolo, V.; Dominguez Rubio, A. P.; Dominici, M.; Dourado, M. R.; Driedonks, T. A.; Duarte, F. V; Duncan, H. M.; Eichenberger, R. M.; Ekström, K.; EL Andaloussi, S.; Elie-Caille, C.; Erdbrügger, U.; Falcón-Pérez, J. M.; Fatima, F.; Fish, J. E.; Flores-Bellver, M.; Försönits, A.; Frelet-Barrand, A.; Fricke, F.; Fuhrmann, G.; Gabrielsson, S.; Gámez-Valero, A.; Gardiner, C.; Gärtner, K.; Gaudin, R.; Gho, Y. S.; Giebel, B.; Gilbert, C.; Gimona, M.; Giusti, I.; Goberdhan, D. C.; Görgens, A.; Gorski, S. M.; Greening, D. W.; Gross, J. C.; Gualerzi, A.; Gupta, G. N.; Gustafson, D.; Handberg, A.; Haraszti, R. A.; Harrison, P.; Hegyesi, H.; Hendrix, A.; Hill, A. F.; Hochberg, F. H.; Hoffmann, K. F.; Holder, B.; Holthofer, H.; Hosseinkhani, B.; Hu, G.; Huang, Y.; Huber, V.; Hunt, S.; Ibrahim, A. G.-E.; Ikezu, T.; Inal, J. M.; Isin, M.; Ivanova, A.; Jackson, H. K.; Jacobsen, S.; Jay, S. M.; Jayachandran, M.; Jenster, G.; Jiang, L.; Johnson, S. M.; Jones, J. C.; Jong, A.; Jovanovic-Talisman, T.; Jung, S.; Kalluri, R.; Kano, S.; Kaur, S.; Kawamura, Y.; Keller, E. 
T.; Khamari, D.; Khomyakova, E.; Khvorova, A.; Kierulf, P.; Kim, K. P.; Kislinger, T.; Klingeborn, M.; Klinke, D. J.; Kornek, M.; Kosanović, M. M.; Kovács, Á. F.; KrämerAlbers, E.-M.; Krasemann, S.; Krause, M.; Kurochkin, I. V; Kusuma, G. D.; Kuypers, S.; Laitinen, S.; Langevin, S. M.; Languino, L. R.; Lannigan, J.; Lässer, C.; Laurent, L. C.; Lavieu, G.; Lázaro-Ibáñez, E.; Le Lay, S.; Lee, M.-S.; Lee, Y. X. F.; Lemos, D. S.; Lenassi, M.; Leszczynska, A.; Li, I. T.; Liao, K.; Libregts, S. F.; Ligeti, E.; Lim, R.; Lim, S. K.; Linē, A.; Linnemannstöns, K.; Llorente, A.; Lombard, C. A.; Lorenowicz, M. J.; Lörincz, Á. M.; Lötvall, J.; Lovett, J.; Lowry, M. C.; Loyer, X.; Lu, Q.; Lukomska, B.; Lunavat, T. R.; Maas, S. L.; Malhi, H.; Marcilla, A.; Mariani, J.; Mariscal, J.; Martens-Uzunova, E. S.; MartinJaular, L.; Martinez, M. C.; Martins, V. R.; Mathieu, M.; Mathivanan, S.; Maugeri, M.; McGinnis, L. K.; McVey, M. J.; Meckes, D. G.; Meehan, K. L.; Mertens, I.; Minciacchi, V. R.; Möller, A.; Møller Jørgensen, M.; Morales-Kastresana, A.; Morhayim, J.; Mullier, F.; Muraca, M.; Musante, L.; Mussack, V.; Muth, D. C.; Myburgh, K. H.; Najrana, T.; Nawaz, M.; Nazarenko, I.; Nejsum, P.; Neri, C.; Neri, T.; Nieuwland, R.; Nimrichter, L.; Nolan, J. P.; Nolte-’t Hoen, E. N.; Noren Hooten, N.; O’Driscoll, L.; O’Grady, T.; O’Loghlen, A.; Ochiya, T.; Olivier, M.; Ortiz, A.; Ortiz, L. A.; Osteikoetxea, X.; Østergaard, O.; Ostrowski, M.; Park, J.; Pegtel, D. M.; Peinado, H.; Perut, F.; Pfaffl, M. W.; Phinney, D. G.; Pieters, B. C.; Pink, R. C.; Pisetsky, D. S.; Pogge von Strandmann, E.; Polakovicova, I.; Poon, I. K.; Powell, B. H.; Prada, I.; Pulliam, L.; Quesenberry, P.; Radeghieri, A.; Raffai, R. L.; Raimondo, S.; Rak, J.; Ramirez, M. I.; Raposo, G.; Rayyan, M. S.; Regev-Rudzki, N.; Ricklefs, F. L.; Robbins, P. D.; Roberts, D. D.; Rodrigues, S. C.; Rohde, E.; Rome, S.; Rouschop, K. M.; Rughetti, A.; Russell, A. E.; Saá, P.; Sahoo, S.; Salas-Huenuleo, E.; Sánchez, C.; Saugstad, J. A.; Saul, M. J.; Schiffelers, R. M.; Schneider, R.; Schøyen, T. H.; Scott, A.; Shahaj, E.; Sharma, S.; Shatnyeva, O.; Shekari, F.; Shelke, G. V.; Shetty, A. K.; Shiba, K.; Siljander, P. R.-M.; Silva, A. M.; Skowronek, A.; Snyder, O. L.; Soares, R. P.; Sódar, B. W.; Soekmadji, C.; Sotillo, J.; Stahl, P. D.; Stoorvogel, W.; Stott, S. L.; Strasser, E. F.; Swift, S.; Tahara, H.; Tewari, M.; Timms, K.; Tiwari, S.; Tixeira, R.; Tkach, M.; Toh, W. S.; Tomasini, R.; Torrecilhas, A. C.; Tosar, J. P.; Toxavidis, V.; Urbanelli, L.; Vader, P.; van Balkom, B. W.; van der Grein, S. G.; Van Deun, J.; van Herwijnen, M. J.; Van KeurenJensen, K.; van Niel, G.; van Royen, M. E.; van Wijnen, A. J.; Vasconcelos, M. H.; Vechetti, I. J.; Veit, T. D.; Vella, L. J.; Velot, É.; Verweij, F. J.; Vestad, B.; Viñas, J. L.; Visnovitz, T.; Vukman, K. V; Wahlgren, J.; Watson, D. C.; Wauben, M. H.; Weaver, A.; Webber, J. P.; Weber, V.; Wehman, A. M.; Weiss, D. J.; Welsh, J. A.; Wendt, S.; Wheelock, A. M.; Wiener, Z.; Witte, L.; Wolfram, J.; Xagorari, A.; Xander, P.; Xu, J.; Yan, X.; Yáñez-Mó, M.; Yin, H.; Yuana, Y.; Zappulli, V.; Zarubova, J.; Žèkas, V.; Zhang, J.; Zhao, Z.; Zheng, L.; Zheutlin, A. R.; Zickler, A. M.; Zimmermann, P.; Zivkovic, A. M.; Zocco, D.; Zuba-Surma, E. K.: Minimal information for studies of extracellular vesicles 2018 (MISEV2018): a position statement of the International Society for Extracellular Vesicles and update of the MISEV2014 guidelines. Journal of Extracellular Vesicles, 7, 1535750 (2018). http://doi:10.1080/20013078.2018.1535750.

[28] Pirri, G.; Damin, F.; Chiari, M.; Bontempi, E.; Depero, L. E.: Characterization of A Polymeric Adsorbed Coating for DNA Microarray Glass Slides. Analytical Chemistry, 76, 1352-1358 (2004). http://doi:10.1021/ac0352629.

[29] Cvjetkovic, A.; Jang, S. C.; Konečná, B.; Höög, J. L.; Sihlbom, C.; Lässer, C.; Lötvall, J.: Detailed Analysis of Protein Topology of Extracellular Vesicles-Evidence of Unconventional Membrane Protein Orientation. Scientific Reports, (2016). http://doi:10.1038/srep36338.

[30] Skliar, M.; Chernyshev, V. S.; Belnap, D. M.; Sergey, G. V.; Al-Hakami, S. M.; Bernard, P. S.; Stijleman, I. J.; Rachamadugu, R.: Membrane proteins significantly restrict exosome mobility. Biochemical and Biophysical Research Communications, 501, 1055-1059 (2018). http://doi:10.1016/j.bbrc.2018.05.107.

[31] Özkumur, E.; Yalcin, A.; Cretich, M.; Damin, F.; Lopez, C.; Bergstein, D. A.; Goldberg, B. 
B.; Chiari, M.; Ünlü, M. S.: Optical phase to biological mass conversion for label-free interferometric sensing methods. In Conference Proceedings - Lasers and Electro-Optics Society Annual Meeting-LEOS; (2009)

[32] Cretich, M.; Monroe, M. R.; Reddington, A.; Zhang, X.; Daaboul, G. G.; Damin, F.; Sola, L.; Unlu, M. S.; Chiari, M.: Interferometric silicon biochips for label and label-free DNA and protein microarrays. Proteomics, 12, 2963-2977 (2012).

http://doi:10.1002/pmic.201200202.

\section{Materials and Methods}

\section{Reagents}

Reagents for peptide synthesis were from Iris Biotech (Marktredwitz, Germany). Other chemicals were from Sigma-Aldrich (St. Louis, MO, USA) if not stated otherwise.

Bare silicon chips and Tetraspanin Kits (chips spotted with anti CD9/CD63/CD81 antibodies) were provided by NanoView Biosciences (Bostom MA). MCP-6 azido copolymer was obtained from Lucidant Polymers (\#MCP-6; Sunnyvale, CA, USA; www.lucidant.com) qEVsingle Size Exclusion Column kit was from Izon Science (Oxford, UK).

\section{Isolation of HEK EVs by ultracentrifugation}

Three days conditioned media from HEK cells were harvested and centrifuged at $500 \mathrm{~g}$ for 25 minutes. Supernatants were filtered with $0.22 \mathrm{~mm}$ filters (Merck Millipore) and centrifuged in a Sorvall ${ }^{\mathrm{TM}}$ WX Ultracentrifuge (ThermoFisher Scientific, WX Ultra 100 \#75000100) at $150.000 \mathrm{~g}$ for 90 minutes at $4^{\circ} \mathrm{C}$ with a SureSpin ${ }^{\mathrm{TM}} 630$ swinging bucket rotor (ThermoFisher Scientific) to pellet EVs. After supernatant was carefully removed, EVs-containing pellet were resuspended in PBS and stored at $-80^{\circ} \mathrm{C}$ until use.

\section{Serum separation}

Four mL of blood were collected in BD VACUTAINER (clot activator tube). Serum samples were separated after centrifugation within two hours from blood collection at $1900 \mathrm{~g}$ for 10 minutes at $4^{\circ} \mathrm{C}$. Serum samples from 5 healthy controls were pooled and frozen at $-20^{\circ} \mathrm{C}$ until use.

\section{Isolation of serum EVs by ultracentrifugation}

$1 \mathrm{~mL}$ of serum pool was filtered with $0.22 \mathrm{~mm}$ filters (Merck Millipore) diluted 1:1 with PBS and centrifuged in a Optima ${ }^{\mathrm{TM}}$ TLX Preparative Ultracentrifuge, Beckman CoulterTM at $150.000 \mathrm{~g}$ for 120 minutes at $4^{\circ} \mathrm{C}$ with a TLA-55 Rotor (Beckman CoulterTM) to pellet EVs. After supernatant was carefully removed, EVs-containing pellet were stored at $-80^{\circ} \mathrm{C}$ until use.

\section{EV isolation by size exclusion chromatography (SEC)}

SEC was performed according to the manufacturer's instructions of the qEV single column (Izon, Christchurch, New Zealand). Briefly, after column equilibration with PBS, $150 \mu \mathrm{L}$ of the pool of sera were loaded, $200 \mu \mathrm{L}$ were collected; the first 5 fractions (F1-F5) were discarded. The seventh fraction (F7) that according to the manufacturer represents the fraction with the highest amount of vesicles was used for analysis and trypsin treatment.

\section{Trypsin treatment}

F7 SEC sample was incubated with $25 \mu \mathrm{g} / \mathrm{mL}$ Trypsin for 6 hours at $37^{\circ} \mathrm{C}$ in a Eppendorf Thermomixer according to published protocols [29], [30]. 


\section{Nanoparticle Tracking Analysis (NTA)}

Nanoparticle tracking analysis (NTA) was performed according to manufacturer's instructions using a NanoSight NS300 system (Malvern Technologies, Malvern, UK) configured with $532 \mathrm{~nm}$ laser. All samples were diluted in filtered PBS to a final volume of $1 \mathrm{ml}$. Ideal measurement concentrations were found by pre-testing the ideal particle per frame value (20-100 particles/frame). Following settings were set according to the manufacturer's software manual. A syringe pump with constant flow injection was used and three videos of $60 \mathrm{~s}$ were captured and analysed with NTA software version 3.2. From each video, the mean, mode, and median EVs size was used to calculate samples concentration expressed in nanoparticles $/ \mathrm{mL}$.

\section{Transmission Electron Microscopy (TEM)}

Isolated EVs were absorbed on glow discharged carbon coated formvar copper grids, washed with water, contrasted with 2\% uranyl acetate and air-dried. Grids were observed with a Zeiss LEO 512 transmission electron microscope. Images were acquired by a $2 \mathrm{k}$ x $2 \mathrm{k}$ bottom-mounted slow-scan Proscan camera controlled by EsivisionPro 3.2 software.

\section{Western Blot analysis}

Purified EVs were resuspended in not reducing Laemmli buffer for the detection of CD9 and CD63, in reducing buffer for ALIX and TSG101 and boiled for 5 minutes at $95^{\circ} \mathrm{C}$. Proteins were resolved by SDS-PAGE and electro-transferred onto a nitrocellulose membrane. Nonspecific sites were blocked with $5 \%(\mathrm{w} / \mathrm{v})$ skimmed milk in T-TBS (Tris-buffered saline: $150 \mathrm{mM} \mathrm{NaCl}, 20 \mathrm{mM} \mathrm{TrisHCl}$, $\mathrm{pH} 7.4$, and $0.5 \%$ Tween 20). Membranes were incubated overnight at $4^{\circ} \mathrm{C}$ with the following antibodies: mouse anti-CD9 (1:5000, BD Pharmingen, \#555370, San Jose, CA, USA), mouse antiCD63 (1:20000; BD Pharmingen, \#556019, San Jose, CA, USA), mouse anti-ALIX (1:500, Santa Cruz, \#sc-271975, Santa Cruz, CA, USA) and mouse anti-TSG101 (1:500, Novus Bio, \#NB200-112, Littleton, CO, USA). After washing with T-TBS, membranes were incubated with goat anti-mouse (1:10000-1:50000) IgG conjugated to horse-radish peroxidase for $45 \mathrm{~min}$. Positive immunoreactive bands were detected by the enhanced chemiluminescence method (Immobilon ${ }^{\mathrm{TM}}$ HRP substrate, \#WBKLS0500, Millipore Corp., Billerica, MA, USA).

\section{Coating of microarray silicon chips with MCP-6}

Silicon chips were coated according to the protocol described in [26]. Briefly, chips were immersed in a MCP-6 solution ( $1 \% \mathrm{w} / \mathrm{v}$ in $\left.0.9 \mathrm{M}\left(\mathrm{NH}_{4}\right)_{2} \mathrm{SO}_{4}\right)$, filtered at $200 \mu \mathrm{m}$, for $30 \mathrm{~min}$. The chips were then rinsed with Milli-Q water and cured for $15 \mathrm{~min}$ at $80^{\circ} \mathrm{C}$.

\section{Peptide synthesis and characterization}

Peptides were assembled by stepwise microwave-assisted Fmoc-SPPS on a Biotage ALSTRA Initiator+ peptide synthesizer, operating in a $0.05 \mathrm{mmol}$ scale. Activation of entering Fmoc-protected amino acids (0.3M solution in DMF) was performed using 0.5 M Oxyma in DMF / 0.5 M DIC in DMF (1:1:1 molar ratio), with a 5 equivalent excess over the initial resin loading. For the linear form BP and BPn coupling steps were performed for 20 minutes at $50^{\circ} \mathrm{C}$. For the branched and tandem forms (BPb and $\mathbf{B P t}$ ) coupling steps were performed for 45 minutes at $50^{\circ} \mathrm{C}$. Chain ramification for the branched $\mathrm{BPb}$ was introduced by coupling Fmoc-Lys(Fmoc)-OH to the growing peptide chain. Capping steps were performed by treatment with a $0.3 \mathrm{M} \mathrm{Ac}_{2} \mathrm{O} / 0.3 \mathrm{M}$ DIEA solution in DMF $(1 \mathrm{x}$ $5 \mathrm{~min})$. Fmoc- deprotection steps were performed by treatment with a $20 \%$ piperidine solution in DMF at room temperature (1 x $10 \mathrm{~min})$. Following each coupling, capping or deprotection step, peptidyl-resin was washed with DMF $(3 \times 3.5 \mathrm{~mL})$. Upon complete chain assembly, resin was washed with DCM ( 5 x $3.5 \mathrm{~mL}$ ) and gently dried under nitrogen flow. Resin-bound peptide was treated with 
an ice-cold TFA, TIS, water, thioanisole mixture (90:5:2.5:2.5 v/v/v/v, 3mL). After gently shaking the resin for 2 hours at room temperature, the resin was filtered and washed with neat TFA ( 2 x 4 $\mathrm{mL}$ ). Cleavage mixture was concentrated under nitrogen stream and then added dropwise to ice-cold diethyl ether $(40 \mathrm{~mL})$ to precipitate the crude peptide. The crude peptide was collected by centrifugation and washed with further cold diethyl ether to remove scavengers. Peptide was then dissolved in $0.1 \%$ TFA aqueous buffer (with minimal addition of ACN to aid dissolution, if necessary). Residual diethyl ether was removed by a gentle nitrogen stream and the crude peptide was purified by RP-HPLC and pure fractions combined and analysed by ESI-MS.

\section{Peptide microarrays}

Microarrays were arrayed on MCP-6 coated patterned silicon chips, with $80 \mathrm{~nm}$ oxide layer thickness, using a non-contact S12 Spotter (Scienion Co., Berlin, Germany), depositing 1 drop for each spot.

Peptides were first dissolved in DMSO to $1 \mathrm{mM}$ stock solution and then diluted to the final spotting concentration $(100 \mu \mathrm{M})$ into the printing buffer for CuACC conjugation on MCP-6 coated surfaces (5 mM Na/Acetate pH 4.8, 50 mM Trehalose, $100 \mu \mathrm{M}$ CuSO4, $400 \mu \mathrm{M}$ THPTA and $6.25 \mathrm{mM}$ Ascorbic Acid).

Printed chips were placed in a humid chamber and incubated overnight at room temperature. The following day chips were immersed in a filtered 2 mM EDTA water solution for $1 \mathrm{~h}$, then washed with Milli-Q water and dried.

Probe density for each immobilized peptide on the array was assessed using label-free film thickness measurement using on the Nanoview platform by nanoQC2.3.2-IT software (NanoView Biosciences, Boston, MA) and previously determined calibration factors for IRIS (Interferometric Reflectance Imaging Sensor) [31], [32]. The average amount of each immobilized peptide was 1.5$3.5 \mathrm{ng} / \mathrm{mm}^{2}$.

\section{EV analyses with ExoView}

EV samples were diluted in filtered PBS and incubated for 2 hours and 30 minutes in static conditions on the printed chips in a humid chamber. EV label-free analysis were carried out using the ExoView R100 reader (NanoView Biosciences, Boston, MA). The reader automatically acquires interferometric images of the microarray. NanoViewer 2.6.0 software counts nanoparticles captured on the peptide spots within a user defined particle contrast.

For fluorescence immune-staining, samples were diluted in incubation buffer(NanoView Biosciences). The samples were incubated on the ExoView Tetraspanin Chip (EV-TC-TTS-01) placed in a sealed 24-well plate for $16 \mathrm{~h}$ at room temperature. The chips were then washed three times in $750 \mu \mathrm{L}$ of incubation buffer for 3 min each on an orbital shaker. Then, chips were incubated with ExoView Tetraspanin Labelling ABs (EV-TC-AB-01) that consist of anti-CD81 Alexa-555, anti-CD63 Alexa-488, and anti-CD9 Alexa-647. The antibodies were diluted 1:5000 in incubation buffer with $2 \%$ BSA. The chips were incubated with $500 \mu \mathrm{L}$ of the labelling solution for $2 \mathrm{~h}$. The chips were then washed once in incubation buffer, three times in wash buffer followed by a rinse in filtered DI water and dried. The chips were then imaged with the ExoView R100 reader using the nScan 2.8.4 acquisition software. The data were then analysed using NanoViewer 2.8.4 
Peptide microarrays were also prepared on gold SPRi chips, purchased from Horiba Scientific SAS (SPRi-Biochip), following the same protocol described above. XelPleX instrument (Horiba Scientific SAS) was firstly calibrated with a solution of $3 \mathrm{mg} / \mathrm{ml}$ of sucrose and then $500 \mu \mathrm{l}$ of serum sample (diluted 1:10 in running buffer) were injected on the surface of the chip with a flow rate of $10 \mu \mathrm{l} / \mathrm{min}$. Subsequently, $200 \mu \mathrm{l}$ of a mixture of anti-human CD9/CD63/CD81 antibodies (500 nM each), purchased from Ancell Corporation, were injected at $25 \mu \mathrm{l} / \mathrm{min}$ in order to confirm the presence of EVs immobilized on the chip. EzSuite and OriginLab softwares were used to analyze the SPRi signals related to each injection.

We have submitted all relevant data of our experiments to the EV-TRACK knowledgebase (EVTRACK ID: EV190066) (Van Deun J, et al. EV-TRACK: transparent reporting and centralizing knowledge in extracellular vesicle research. Nature methods. 2017;14(3):228-32)." 